Geneste, P., Kamenka, J. M., Roques, R., Declerce, J.-P. \& Germain, G. (1981). Tetrahedron Lett. 22, 949-950.

Jones, L. A., Beaver, R. W., SChmoeger, T. L., Ort, J. F. \& Leander, J. D. (1981). J. Org. Chem. 46, 3330-3333.

Kimura, M. \& OKabayashi, I. (1986). J. Heterocycl. Chem. 23, 1287-1289.

Nelson, H. C. \& Simonsen, S. H. (1981). Acta Cryst. A37, $\mathrm{Cl} 68$.

Acta Cryst. (1991). C47, 225-227
SHELDRICK, G. M. (1985), SHELXTL User's Manual. Revision 5.1. Nicolet XRD Corporation, Madison, Wisconsin, USA.

Singh, P., Jones, L. A. \& CouCh, R. (1988). Acta Cryst. C44, 1976-1979.

SPARKS, R. A. (1961). In Computing Methods and the Phase Problem in X-ray Crystal Analysis, edited by R. A. PEPINSKY, J. M. Robertson \& J. C. Speakman, pp. 170-187. New York: Pergman Press.

\title{
Complexation of Water by a 4-Phenylpyridohemispherand
}

\author{
By Pieter Jelle Dijkstra, Herman Johannes Den Hertog Jr and David Nicolaas Reinhoudt \\ Laboratory of Organic Chemistry, University of Twente, PO Box 217, 7500 AE Enschede, The Netherlands \\ and Sybolt HaRkema \\ Laboratory of Chemical Physics, University of Twente, PO Box 217, 7500 AE Enschede, The Netherlands
}

(Received 9 February 1990; accepted 18 June 1990)

\begin{abstract}
Dimethoxy-9,23-dimethyl-4-phenyl$13,16,19$-trioxa-27-azoniatetracyclo[19.3.1.1 $\left.1^{2,6} \cdot 1^{7.11}\right]$ heptacosa-1(25),2,4,6(27), 7,9,11(26),21,23-nonaene perchlorate-ethanol-water $(1 / 1 / 1), \quad \mathrm{C}_{33} \mathrm{H}_{36} \mathrm{NO}_{5}^{+}$.$\mathrm{ClO}_{4}^{-} . \mathrm{C}_{2} \mathrm{H}_{5} \mathrm{OH} . \mathrm{H}_{2} \mathrm{O}, \quad M_{r}=690 \cdot 2$, orthorhombic, $P_{c a 2_{1}}, a=27.747$ (5), $b=9.572(3), c=12.866$ (4) $\AA$, $V=3417(3) \AA^{3}, Z=4, D_{x}=1 \cdot 39 \mathrm{~g} \mathrm{~cm}^{-3}, \lambda($ Mo $K \alpha)$ $=0.7107 \AA, \quad \mu=1.69 \mathrm{~cm}^{-1}, \quad F(000)=1464, \quad T=$ $100(5) \mathrm{K}$, final $R=6 \cdot 5 \%$ for 2915 observed reflections. The water molecule is complexed in the cavity of the macrocycle with hydrogen bonds to the polyethylene oxy atoms adjacent to the outer phenyl rings of the terphenyl unit. The $\mathrm{O}$ atom of the water molecule accepts hydrogen bonds from the pyridinium proton and the $\mathrm{OH}$ proton of the ethanol molecule. The $\mathrm{HClO}_{4}$ proton is transferred to the pyrido nitrogen.
\end{abstract}

Experimental. The title compound was prepared by dissolving $50 \mathrm{mg}(0.09 \mathrm{mmol})$ of the 4-phenylpyridohemispherand (Dijkstra, den Hertog, van Steen, Zijlstra, Skowronska-Ptasinska, Reinhoudt, van Eerden \& Harkema, 1987) in $2 \mathrm{ml}$ of ethanol and the slow addition of $0.5 \mathrm{ml}$ of $70 \% \mathrm{HClO}_{4}$. Slow evaporation of solvent afforded colorless crystals of the complex suitable for X-ray diffraction, m.p. $496 \mathrm{~K}$ (dec.).

Intensities were measured at $100(5) \mathrm{K}$ on an Enraf-Nonius CAD-4 diffractometer using graphitemonochromated Mo $K \alpha$ radiation $(\lambda=0.7107 \AA)$. Lattice parameters determined by least squares from 25 centered reflections $6<\theta<13^{\circ}$. A total of 2915 independent reflections up to $\theta=25^{\circ}(0 \leq h \leq 33,0$

0108-2701/91/010225-03\$03.00 $\leq k \leq 12,0 \leq l \leq 16)$ were measured in the $\theta / 2 \theta$ scan mode [scan speed $0.046^{\circ} \mathrm{s}^{-1}$, scan width $(\theta) 1.5^{\circ}$ ]; 2021 considered observed $\left[F_{o}^{2}>2 \sigma\left(F_{o}^{2}\right)\right]$. The intensity loss of three standard reflections measured every hour was less than $1 \cdot 3 \%$, correction for which was applied. No absorption correction.

$\mathrm{H}$ atoms of the macrocycle were placed in calculated positions and treated as riding on their parent atoms (bond distance $0.95 \AA, B_{\text {iso }}=4.0 \AA^{2}$ ), with the exception of the methyl hydrogens. These atoms and the $\mathrm{H}$ atoms of the water molecule were found from a difference Fourier synthesis. Positions of these atoms were refined. Hydrogens of the ethanol group could not be located (probably due to the large thermal motions). These hydrogens were not included in the refinement. In order to fix the origin in the $c$ direction, the $z$ parameter of the $\mathrm{Cl}$ atom has been fixed.

The structure was solved with MULTAN (Germain, Main \& Woolfson, 1971) and refined by full-matrix least squares. Weights for each reflection in the refinement (on $F$ ) were calculated from $w=$ $4 F_{o}^{2} / \sigma^{2}\left(F_{o}^{2}\right), \sigma^{2}\left(F_{o}^{2}\right)=\sigma^{2}(I)+\left(p F_{o}^{2}\right)^{2}$; the value of the instability factor $p$ was determined as 0.04 . The number of parameters refined was 457: scale factor, isotropic extinction factor [final value $2.4(7) \times 10^{-9}$ ], positional and anisotropic thermal parameters for the non-H atoms positional parameters of some $\mathrm{H}$ atoms.

Refinement converged at $R=6.5 \%, w R=6.3 \%$, $S=1 \cdot 64,(\Delta / \sigma)_{\max }=0 \cdot 2$. Largest peak on the final difference Fourier map $0.54 \mathrm{e}^{-3}$ (in the perchlorate ion). All calculations were performed using $S D P$

(C) 1991 International Union of Crystallography 
Table 1. Fractional atomic coordinates and equivalent isotropic thermal parameters for non- $\mathrm{H}$ atoms

$$
B_{\mathrm{eq}}=\left(8 \pi^{2} / 3\right) \sum_{i} \sum_{j} U_{i j} a_{i}^{*} a_{j}^{*} \mathbf{a}_{i} \cdot \mathbf{a}_{j}
$$

\begin{tabular}{|c|c|c|c|c|}
\hline & $x$ & $y$ & $z$ & $B_{\text {eq }}\left(\AA^{2}\right)$ \\
\hline $\mathrm{Cl}$ & $0.08729(7)$ & 0.0049 (2) & 0.3979 & $2.25(3)$ \\
\hline 07 & 0.3167 (2) & $0 \cdot 2590(6)$ & $0.5637(4)$ & 2.4 (1) \\
\hline $\mathrm{O} 14$ & $0.3607(2)$ & $0.4576(5)$ & 0.4297 (4) & $2 \cdot 6(1)$ \\
\hline $\mathrm{O} 28$ & $0.4206(2)$ & $0.4044(5)$ & $0.6275(4)$ & $2 \cdot 3(1)$ \\
\hline $\mathrm{O} 32$ & $0.4642(2)$ & $0.4180(5)$ & $0.4088(4)$ & $2 \cdot 3(1)$ \\
\hline O35 & $0.4041(2)$ & $0 \cdot 2064(6)$ & $0.3148(4)$ & $2 \cdot 4(1)$ \\
\hline $\mathrm{O} 38$ & $0 \cdot 3003(2)$ & $0 \cdot 2123(6)$ & $0.3430(4)$ & $2.4(1)$ \\
\hline O51 & $0.0822(2)$ & $-0.1355(6)$ & $0.4297(5)$ & $3 \cdot 2(1)$ \\
\hline O52 & $0.1299(2)$ & $0.0152(6)$ & $0.3336(5)$ & $2 \cdot 9(1)$ \\
\hline $\mathrm{O} 53$ & $0.0460(3)$ & $0.0430(8)$ & $0.3406(6)$ & $5.0(2)$ \\
\hline 054 & $0.0919(2)$ & $0.0947(8)$ & $0.4854(6)$ & $5.9(2)$ \\
\hline 0100 & $0.3668(2)$ & $0.6430(7)$ & $0.2616(5)$ & $4.0(2)$ \\
\hline N1l & $0.3272(2)$ & $0.5247(6)$ & $0.6522(5)$ & $1.5(1)$ \\
\hline $\mathrm{Cl}$ & $0.2416(3)$ & $0 \cdot 2442(8)$ & $0.4741(7)$ & $2 \cdot 1(2)$ \\
\hline $\mathrm{C} 2$ & 0.1947 (3) & $0.2874(8)$ & $0.4648(7)$ & $2.5(2)$ \\
\hline $\mathrm{C} 3$ & $0 \cdot 1736(3)$ & $0.3904(9)$ & $0.5273(7)$ & $2 \cdot 7(2)$ \\
\hline C4 & $0.2034(3)$ & $0.4525(8)$ & $0.6018(7)$ & $2 \cdot 3(2)$ \\
\hline C5 & $0.2517(3)$ & $0.4121(7)$ & $0.6134(6)$ & $1.6(2)$ \\
\hline C6 & $0 \cdot 2700(3)$ & $0 \cdot 3046(8)$ & $0.5532(6)$ & $1.9(2)$ \\
\hline $\mathrm{C} 8$ & $0.3271(3)$ & $0.1850(8)$ & $0.6561(7)$ & $3 \cdot 1(2)$ \\
\hline $\mathrm{C} 9$ & $0.1218(3)$ & $0.4382(10)$ & $0.5141(7)$ & $3 \cdot 1(2)$ \\
\hline $\mathrm{ClO}$ & $0.2823(3)$ & $0.4966(8)$ & $0.6844(6)$ & $1.7(2)$ \\
\hline $\mathrm{C} 12$ & $0.3565(3)$ & $0.6150(8)$ & $0.7032(6)$ & $1.7(2)$ \\
\hline $\mathrm{C} 13$ & $0.3396(3)$ & $0.6754(8)$ & $0.7937(6)$ & $1.8(2)$ \\
\hline $\mathrm{Cl} 4$ & $0.2933(3)$ & $0.6491(8)$ & $0.8300(6)$ & $1.6(2)$ \\
\hline $\mathrm{Cls}$ & $0.2650(3)$ & $0.5527(8)$ & $0.7740(6)$ & $1.8(2)$ \\
\hline $\mathrm{C} 16$ & $0.2748(3)$ & $0.7180(8)$ & $0.9230(6)$ & $1.8(2)$ \\
\hline $\mathrm{Cl} 7$ & 0.3057 (3) & $0.7668(8)$ & $1.0021(7)$ & $2 \cdot 4(2)$ \\
\hline $\mathrm{Cl} 8$ & $0 \cdot 2873(3)$ & $0.8242(8)$ & $1.0930(7)$ & $2 \cdot 6(2)$ \\
\hline $\mathrm{Cl9}$ & $0 \cdot 2387$ (3) & 0.8407 (8) & $1 \cdot 1045(7)$ & $2 \cdot 8(2)$ \\
\hline $\mathrm{C} 20$ & $0 \cdot 2081(3)$ & $0.8019(8)$ & $1.0285(7)$ & $3 \cdot 1(2)$ \\
\hline $\mathrm{C} 21$ & $0 \cdot 2256(3)$ & $0.7414(8)$ & $0.9370(7)$ & $2.5(2)$ \\
\hline $\mathrm{C} 22$ & $0.4029(3)$ & $0.6479(8)$ & $0.6531(6)$ & $1.8(2)$ \\
\hline $\mathrm{C} 23$ & $0.4165(3)$ & $0.7864(8)$ & $0 \cdot 6401(6)$ & $1.8(2)$ \\
\hline $\mathrm{C} 24$ & $0.4558(3)$ & $0.8237(9)$ & $0.5802(7)$ & $2.5(2)$ \\
\hline $\mathrm{C} 25$ & $0.4819(3)$ & $0.7189(8)$ & $0.5339(7)$ & $2 \cdot 2(2)$ \\
\hline $\mathrm{C} 26$ & $0 \cdot 4706(3)$ & $0.5781(8)$ & $0.5470(6)$ & $1.9(2)$ \\
\hline $\mathrm{C} 27$ & $0.4317(3)$ & $0.5427(8)$ & $0.6120(7)$ & $2.0(2)$ \\
\hline C29 & $0.4391(4)$ & $0.3585(9)$ & $0.7262(7)$ & $3.5(2)$ \\
\hline C30 & $0.4693(3)$ & $0.9760(9)$ & $0.5657(7)$ & $3.4(2)$ \\
\hline C31 & $0.4961(3)$ & $0.4628(9)$ & $0.4885(7)$ & $3.0(2)$ \\
\hline C33 & $0.4821(3)$ & $0.2928(9)$ & $0.3580(7)$ & $2 \cdot 8(2)$ \\
\hline C34 & $0.4488(3)$ & $0.2556(9)$ & $0.2729(7)$ & $2.8(2)$ \\
\hline C36 & $0 \cdot 3696(3)$ & $0 \cdot 1884(9)$ & $0.2350(7)$ & $3.4(2)$ \\
\hline C37 & $0.3257(3)$ & $0 \cdot 1200(10)$ & $0.2764(8)$ & $3.7(2)$ \\
\hline C39 & $0 \cdot 2640(3)$ & $0.1397(8)$ & $0.4035(7)$ & $2.8(2)$ \\
\hline $\mathrm{C} 101$ & $0.4271(5)$ & $0.8201(14)$ & $0.2240(20)$ & $12 \cdot 2(7)$ \\
\hline $\mathrm{C} 102$ & $0.4110(5)$ & $0.7216(16)$ & 0.2789 (17) & $12 \cdot 4(5)$ \\
\hline
\end{tabular}

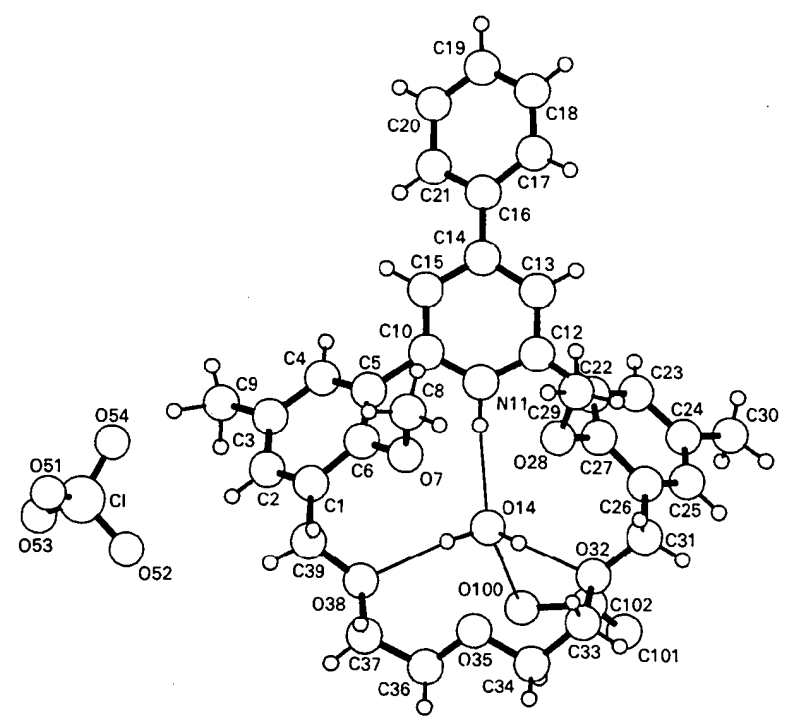

Fig. 1. View of the molecule with the atom-numbering scheme.
Table 2. Bond distances $(\AA)$ and angles $\left(^{\circ}\right)$ for non- $\mathrm{H}$ atoms and torsion angles in the macrocycle $\left({ }^{\circ}\right)$ with e.s.d.'s in parentheses

\begin{tabular}{|c|c|c|c|}
\hline $\mathrm{Cl} \rightleftharpoons \mathrm{O} 51$ & $1 \cdot 413(6)$ & $\mathrm{C} 5-\mathrm{ClO}$ & $1.49(2)$ \\
\hline $\mathrm{Cl}-\mathrm{O} 52$ & $1.446(6)$ & $\mathrm{C} 10-\mathrm{Cl} 15$ & $1 \cdot 36(2)$ \\
\hline $\mathrm{Cl}-\mathrm{O} 53$ & $1.410(7)$ & $\mathrm{C} 12-\mathrm{Cl} 3$ & $1 \cdot 38(2)$ \\
\hline $\mathrm{Cl}-\mathrm{O} 54$ & $1.422(8)$ & $\mathrm{C} 12-\mathrm{C} 22$ & $1.47(2)$ \\
\hline $\mathrm{O} 7-\mathrm{C} 6$ & $1.374(9)$ & $\mathrm{C} 13-\mathrm{C} 14$ & $1 \cdot 39(1)$ \\
\hline $\mathrm{O} 7-\mathrm{C} 8$ & $1.41(2)$ & $\mathrm{Cl} 4-\mathrm{Cl} 5$ & $1.41(2)$ \\
\hline $\mathrm{O} 28-\mathrm{C} 27$ & $1.374(9)$ & $\mathrm{C} 14-\mathrm{Cl} 6$ & $1.47(1)$ \\
\hline O28-C29 & $1.44(2)$ & $\mathrm{C} 16-\mathrm{Cl} 7$ & $1.41(1)$ \\
\hline $\mathrm{O} 32-\mathrm{C} 31$ & $1.42(2)$ & $\mathrm{C} 16-\mathrm{C} 21$ & $1 \cdot 39(1)$ \\
\hline $\mathrm{O} 32-\mathrm{C} 33$ & $1.45(2)$ & $\mathrm{C} 17-\mathrm{Cl} 8$ & $1.39(1)$ \\
\hline $\mathrm{O} 35-\mathrm{C} 34$ & $1.43(2)$ & $\mathrm{C} 18-\mathrm{C} 19$ & $1 \cdot 37(1)$ \\
\hline $\mathrm{O} 35-\mathrm{C} 36$ & $1.42(2)$ & $\mathrm{C} 19-\mathrm{C} 20$ & $1.35(1)$ \\
\hline $\mathrm{O} 38-\mathrm{C} 37$ & $1.42(2)$ & $\mathrm{C} 20-\mathrm{C} 21$ & $1.40(1)$ \\
\hline $\mathrm{O} 38-\mathrm{C} 39$ & $1.46(2)$ & $\mathrm{C} 22-\mathrm{C} 23$ & $1 \cdot 39(2)$ \\
\hline $\mathrm{O} 100-\mathrm{Cl} 02$ & $1.46(2)$ & $\mathrm{C} 22-\mathrm{C} 27$ & $1 \cdot 39(2)$ \\
\hline $\mathrm{N} 11-\mathrm{Cl} 0$ & $1.34(1)$ & $\mathrm{C} 23-\mathrm{C} 24$ & $1.38(1)$ \\
\hline $\mathrm{N} 11-\mathrm{C} 12$ & $1.357(9)$ & $\mathrm{C} 24-\mathrm{C} 25$ & $1.37(1)$ \\
\hline $\mathrm{C} 1-\mathrm{C} 2$ & $1 \cdot 37(1)$ & $\mathrm{C} 24-\mathrm{C} 30$ & $1.52(1)$ \\
\hline $\mathrm{Cl}-\mathrm{C} 6$ & $1.42(1)$ & $\mathrm{C} 25-\mathrm{C} 26$ & $1.39(1)$ \\
\hline $\mathrm{C} 1-\mathrm{C} 39$ & $1.49(1)$ & $\mathrm{C} 26-\mathrm{C} 27$ & $1.41(2)$ \\
\hline $\mathrm{C} 2-\mathrm{C} 3$ & $1.41(1)$ & $\mathrm{C} 26-\mathrm{C} 31$ & $1.51(1)$ \\
\hline $\mathrm{C} 3-\mathrm{C} 4$ & $1.40(1)$ & $\mathrm{C} 33-\mathrm{C} 34$ & $1.48(1)$ \\
\hline $\mathrm{C} 3-\mathrm{C} 9$ & $1.52(1)$ & C $36-C 37$ & $1.48(1)$ \\
\hline $\mathrm{C} 4-\mathrm{C} 5$ & $1.40(2)$ & $\mathrm{C} 101-\mathrm{C} 102$ & $1 \cdot 26(3)$ \\
\hline $\mathrm{C} 5-\mathrm{C} 6$ & $1 \cdot 38(2)$ & & \\
\hline $\mathrm{O} 51-\mathrm{Cl}-\mathrm{O} 52$ & $108 \cdot 2(3)$ & $\mathrm{C} 13-\mathrm{Cl} 4-\mathrm{Cl} 15$ & $117 \cdot 6(8)$ \\
\hline $\mathrm{O} 51-\mathrm{Cl}-\mathrm{O} 53$ & $108.4(5)$ & $\mathrm{C} 13-\mathrm{C} 14-\mathrm{Cl} 6$ & $121 \cdot 3(7)$ \\
\hline $\mathrm{O} 51-\mathrm{Cl}-\mathrm{O} 54$ & $110.8(5)$ & $\mathrm{Cl} 5-\mathrm{Cl} 4-\mathrm{Cl} 6$ & $121 \cdot 3(7)$ \\
\hline $\mathrm{O} 52-\mathrm{Cl}-\mathrm{O} 53$ & $110 \cdot 4(4)$ & $\mathrm{C} 10-\mathrm{Cl5}-\mathrm{Cl} 4$ & $119 \cdot 7(7)$ \\
\hline $\mathrm{O} 52-\mathrm{Cl}-\mathrm{O} 54$ & $109 \cdot 8(4)$ & $\mathrm{Cl} 4-\mathrm{Cl} 6-\mathrm{Cl} 7$ & $121 \cdot 8(8)$ \\
\hline $\mathrm{O} 53-\mathrm{Cl}-\mathrm{O} 54$ & $109 \cdot 4(4)$ & $\mathrm{C} 14-\mathrm{C} 16-\mathrm{C} 21$ & $121 \cdot 5(8)$ \\
\hline $\mathrm{C} 6-\mathrm{O} 7-\mathrm{C} 8$ & $115 \cdot 8(6)$ & $\mathrm{Cl} 7-\mathrm{C} 16-\mathrm{C} 21$ & $116 \cdot 7(7)$ \\
\hline $\mathrm{C} 27-\mathrm{O} 28-\mathrm{C} 29$ & $110 \cdot 1(6)$ & $\mathrm{C} 16-\mathrm{C} 17-\mathrm{Cl} 8$ & $120.9(8)$ \\
\hline $\mathrm{C} 31-\mathrm{O} 32-\mathrm{C} 33$ & $111 \cdot 1(6)$ & $\mathrm{Cl} 7-\mathrm{Cl} 8-\mathrm{Cl} 19$ & $120 \cdot 0(8)$ \\
\hline $\mathrm{C} 34-\mathrm{O} 35-\mathrm{C} 36$ & $110 \cdot 7(6)$ & $\mathrm{C} 18-\mathrm{C} 19-\mathrm{C} 20$ & $120 \cdot 8(8)$ \\
\hline $\mathrm{C} 37-\mathrm{O} 38-\mathrm{C} 39$ & $111 \cdot 7(7)$ & $\mathrm{C} 19-\mathrm{C} 20-\mathrm{C} 21$ & $120 \cdot 4(8)$ \\
\hline $\mathrm{Cl}-\mathrm{N} 11-\mathrm{C} 12$ & $122 \cdot 4(6)$ & $\mathrm{C} 16-\mathrm{C} 21-\mathrm{C} 20$ & $120 \cdot 9(8)$ \\
\hline $\mathrm{C} 2-\mathrm{C} 1-\mathrm{C} 6$ & $117.9(7)$ & $\mathrm{C}_{12}-\mathrm{C} 22-\mathrm{C} 23$ & $119 \cdot 6(7)$ \\
\hline $\mathrm{C} 2-\mathrm{C} 1-\mathrm{C} 39$ & $123 \cdot 2(8)$ & $\mathrm{C} 12-\mathrm{C} 22-\mathrm{C} 27$ & $120 \cdot 9(7)$ \\
\hline $\mathrm{C} 6-\mathrm{Cl}-\mathrm{C} 39$ & $119 \cdot 0(7)$ & $\mathrm{C} 23-\mathrm{C} 22-\mathrm{C} 27$ & $119 \cdot 4(8)$ \\
\hline $\mathrm{C} 1-\mathrm{C} 2-\mathrm{C} 3$ & $124 \cdot 0(8)$ & $\mathrm{C} 22-\mathrm{C} 23-\mathrm{C} 24$ & $121 \cdot 9(7)$ \\
\hline $\mathrm{C} 2-\mathrm{C} 3-\mathrm{C} 4$ & $116 \cdot 5(8)$ & $\mathrm{C} 23-\mathrm{C} 24-\mathrm{C} 25$ & $118.0(8)$ \\
\hline $\mathrm{C} 2-\mathrm{C} 3-\mathrm{C} 9$ & $122 \cdot 9(8)$ & $\mathrm{C} 23-\mathrm{C} 24-\mathrm{C} 30$ & $120.7(8)$ \\
\hline $\mathrm{C} 4-\mathrm{C} 3-\mathrm{C} 9$ & $120 \cdot 5(8)$ & $\mathrm{C} 25-\mathrm{C} 24-\mathrm{C} 30$ & $121 \cdot 3(8)$ \\
\hline $\mathrm{C} 3-\mathrm{C} 4-\mathrm{C} 5$ & $121 \cdot 5(7)$ & $\mathrm{C} 24-\mathrm{C} 25-\mathrm{C} 26$ & $122.4(7)$ \\
\hline $\mathrm{C} 4-\mathrm{C} 5-\mathrm{C} 6$ & $119.6(8)$ & $\mathrm{C} 25-\mathrm{C} 26-\mathrm{C} 27$ & $118.5(7)$ \\
\hline $\mathrm{C} 4-\mathrm{C} 5-\mathrm{C} 10$ & $117.5(7)$ & $\mathrm{C} 25-\mathrm{C} 26-\mathrm{C} 31$ & $122 \cdot 7(8)$ \\
\hline $\mathrm{C} 6-\mathrm{C} 5-\mathrm{C} 10$ & $122 \cdot 7(7)$ & $\mathrm{C} 27-\mathrm{C} 26-\mathrm{C} 31$ & $118.7(8)$ \\
\hline $\mathrm{O} 7-\mathrm{C} 6-\mathrm{Cl}$ & $117 \cdot 8(7)$ & $\mathrm{O} 28-\mathrm{C} 27-\mathrm{C} 22$ & $121 \cdot 0(7)$ \\
\hline $\mathrm{O} 7-\mathrm{C} 6-\mathrm{C} 5$ & $121 \cdot 7(7)$ & $\mathrm{O} 28-\mathrm{C} 27-\mathrm{C} 26$ & $119 \cdot 3(7)$ \\
\hline $\mathrm{C} 1-\mathrm{C} 6-\mathrm{C} 5$ & $120 \cdot 3(7)$ & $\mathrm{C} 22-\mathrm{C} 27-\mathrm{C} 26$ & $119 \cdot 5(7)$ \\
\hline $\mathrm{N} 11-\mathrm{ClO}-\mathrm{C} 5$ & $116 \cdot 7(6)$ & $\mathrm{O} 32-\mathrm{C} 31-\mathrm{C} 26$ & $106 \cdot 8(7)$ \\
\hline $\mathrm{N} 11-\mathrm{C} 10-\mathrm{C} 15$ & $120 \cdot 8(7)$ & $\mathrm{O} 32-\mathrm{C} 33-\mathrm{C} 34$ & $108 \cdot 5(7)$ \\
\hline $\mathrm{C} 5-\mathrm{C} 10-\mathrm{C} 15$ & $122 \cdot 4(7)$ & $\mathrm{O} 35-\mathrm{C} 34-\mathrm{C} 33$ & $110 \cdot 1(8)$ \\
\hline $\mathrm{N} 11-\mathrm{C} 12-\mathrm{C} 13$ & $118.0(7)$ & $\mathrm{O} 35-\mathrm{C} 36-\mathrm{C} 37$ & $110.4(7)$ \\
\hline $\mathrm{N} 11-\mathrm{C} 12-\mathrm{C} 22$ & $116 \cdot 6(7)$ & $\mathrm{O} 38-\mathrm{C} 37-\mathrm{C} 36$ & $110.5(7)$ \\
\hline $\mathrm{C} 13-\mathrm{C} 12-\mathrm{C} 22$ & $125 \cdot 2(7)$ & $\mathrm{O} 38-\mathrm{C} 39-\mathrm{Cl}$ & $107 \cdot 1(6)$ \\
\hline $\mathrm{Cl} 2-\mathrm{Cl} 3-\mathrm{Cl} 4$ & $121 \cdot 5(7)$ & $\mathrm{O} 100-\mathrm{C} 102-\mathrm{C} 101$ & $127(2)$ \\
\hline $\mathrm{C} 33-\mathrm{O} 32-\mathrm{C} 31-\mathrm{C} 26$ & $-171 \cdot 1(6)$ & $\mathrm{C} 6-\mathrm{Cl}-\mathrm{C} 39-\mathrm{O} 38$ & $-66 \cdot 1(9)$ \\
\hline $\mathrm{C} 31-\mathrm{O} 32-\mathrm{C} 33-\mathrm{C} 34$ & $-177 \cdot 2(7)$ & $\mathrm{C} 10-\mathrm{C} 5-\mathrm{C} 6-\mathrm{C} 1$ & $-168.7(7)$ \\
\hline $\mathrm{C} 36-\mathrm{O} 35-\mathrm{C} 34-\mathrm{C} 33$ & $172.0(7)$ & $\mathrm{C} 6-\mathrm{C} 5-\mathrm{C} 10-\mathrm{N} 11$ & $36 \cdot 1(11)$ \\
\hline $\mathrm{C} 34-\mathrm{O} 35-\mathrm{C} 36-\mathrm{C} 37$ & $172 \cdot 5(7)$ & $\mathrm{N} 11-\mathrm{C} 12-\mathrm{C} 22-\mathrm{C} 27$ & $-46 \cdot 1(11)$ \\
\hline $\mathrm{C} 3-\mathrm{O} 38-\mathrm{C} 37-\mathrm{C} 36$ & $-166.5(7)$ & $\mathrm{C} 12-\mathrm{C} 22-\mathrm{C} 27-\mathrm{C} 26$ & $167 \cdot 5(7)$ \\
\hline $\mathrm{C} 37-\mathrm{O} 38-\mathrm{C} 39-\mathrm{Cl}$ & $177 \cdot 0(7)$ & $\mathrm{C} 31-\mathrm{C} 26-\mathrm{C} 27-\mathrm{C} 22$ & $-169 \cdot 6(8)$ \\
\hline $\mathrm{C} 12-\mathrm{N} 11-\mathrm{Cl}-\mathrm{C} 5$ & $171 \cdot 4(7)$ & $\mathrm{C} 27-\mathrm{C} 26-\mathrm{C} 31-\mathrm{O} 32$ & $71.9(9)$ \\
\hline $\mathrm{C} 10-\mathrm{N} 11-\mathrm{C} 12-\mathrm{C} 22$ & $-173.5(7)$ & $\mathrm{O} 32-\mathrm{C} 33-\mathrm{C} 34-\mathrm{O} 35$ & $-70 \cdot 1(8)$ \\
\hline $\mathrm{C} 39-\mathrm{Cl}-\mathrm{C} 6-\mathrm{C} 5$ & $173 \cdot 5(7)$ & $\mathrm{O} 35-\mathrm{C} 36-\mathrm{C} 37-\mathrm{O} 38$ & $70 \cdot 1(9)$ \\
\hline
\end{tabular}

Table 3. Hydrogen-bond data $\left(\AA,^{\circ}\right)$

$\begin{array}{lcccccc}\text { Donor } & \text { Hydrogen } & \text { Acceptor } & & & & \\ \text { atom } D & \text { atom H } & \text { atom } A & D-\mathrm{H} & A \cdots \mathrm{H} & D-\mathrm{H} \cdots A & D \cdots A \\ \text { O14 } & \text { H14A } & \text { O38 } & 0.96(7) & 2.13(8) & 174(7) & 2 \cdot 909(7) \\ \text { O14 } & \text { H14B } & \text { O32 } & 0.99(8) & 1.94(8) & 163(7) & 3.093(8) \\ \text { N11 } & \text { H11* } & \text { O14 } & 0.950(7) & 2 \cdot 174(6) & 158 \cdot 4(4) & 3.077(8) \\ \text { O100 } & & \text { O14 } & & & & 2.803(9)\end{array}$

* H11 was placed in a calculated position. Therefore the e.s.d. of bond lengths and angles involving $\mathrm{H} 11$ is determined by the uncertainty in the positions of the heavy atoms only. 
(B. A. Frenz \& Associates, Inc., 1983). Atomic scattering factors from International Tables for X-ray Crystalloraphy (1974, Vol. IV). Atomic parameters of non- $\mathrm{H}$ atoms are in Table $1 .^{*}$ Bond distances and angles and macrocyclic torsion angles are given in Table 2, whereas some important non-bonded distances and hydrogen bonds in the crystal structure of the complex are given in Table 3 . Atom numbering in the macrocycle is shown in Fig. 1.

Related literature. Crystal structures of the uncomplexed 4-pyridohemispherand (Dijkstra, den Hertog, van Eerden, Harkema \& Reinhoudt, 1988), the Na.picrate complex (Dijkstra, den Hertog, van Steen, Zijlstra, Skowronska-Ptasinska, Reinhoudt, van Eerden \& Harkema, 1987) and the complex with malononitrile (van Eerden, Grootenhuis, Dijkstra van Staveren, Harkema \& Reinhoudt, 1986) have been reported.

* Lists of structure factors, anisotropic thermal parameters, $\mathrm{H}$-atom parameters and complete lists of bond lengths and angles have been deposited with the British Library Document Supply Centre as Supplementary Publication No. SUP 53289 (38 pp.). Copies may be obtained through The Technical Editor, International Union of Crystallography, 5 Abbey Square, Chester $\mathrm{CH}$ l $2 \mathrm{HU}$, England.
Upon complexation of water but also with $\mathrm{Na}^{+}$ and malononitrile a rather strong reorganization takes place: in the crystal structure of the uncomplexed ligand the two methoxy groups are on either side of the mean macrocyclic plane, whereby one of the methoxy groups partly converges into the cavity which is partly filled by the methyl groups.

This work was (in part) supported by the Netherlands Foundation for Technical Research (STW) and the Future Technical Science Branch/Division of the Netherlands Organization for the Advancement of Pure Research (ZWO).

\section{References}

B. A. Frenz \& Associates, Inc. (1983). SDP Structure Determination Package. College Station, Texas, USA.

Dijkstra, P. J., den Hertog, H. J. Jr, van Eerden J., Harkema S. \& Reinhoudt, D. N. (1988). J. Org. Chem. 53, 374-382.

Dijkstra, P. J., Den Hertog, H. J. JR, van Steen, B. J., ZiJlstra, S., SKowronska-PTasinSKa, M., Reinhoudt, D. N., van Eerden, J. \& Harkema, S. (1987). J. Org. Chem. 52, 2433-2442.

Eerden, J. van, Grootenhuis, P. D. J., Dijkstra, P. J., van Staveren, C. J., Harkema, S. \& Reinhoudt, D. N. (1986). $J$. Org. Chem. 51, 3918-3920.

Germain, G., Main, P. \& Woolfson, M. M. (1971). Acta Cryst. A27, 368-376.

Acta Cryst. (1991). C47, 227-229

\title{
Structure of 1-(3-Methylphenyl)-4-(2- $\beta$-quinolylethyl)piperazine: Centhaquin
}

\author{
By Alain CARPY \\ URA 605 CNRS, Faculté de Pharmacie, Université de Bordeaux II, 3 Place de la Victoire, \\ 33076 Bordeaux CEDEX, France \\ AND ANIL K. SAXENA \\ Central Drug Research Institute, Chattar Manzil, P. B. $n^{\circ}$ 173, Lucknow-226001, India
}

(Received 14 March 1990; accepted 20 June 1990)

\begin{abstract}
C}_{22} \mathrm{H}_{25} \mathrm{~N}_{3}$, centrally active hypotensive agent, antihypertensive, $M_{r}=331 \cdot 46$, monoclinic, $P 2_{1} / c, a=9 \cdot 122(2), b=22 \cdot 108(3), c=9 \cdot 165(2) \AA$, $\beta=102 \cdot 20(1)^{\circ}, \quad V=1806 \cdot 6 \AA^{3}, \quad Z=4, \quad D_{x}=$ $1.22 \mathrm{~g} \mathrm{~cm}^{-3}, \bar{\lambda}(\mathrm{Cu} K \alpha)=1.54178 \AA, \mu=5.67 \mathrm{~cm}^{-1}$, $F(000)=712$, room temperature, $R=0.043$ for 1360 observed reflections. The methylphenyl substituted nitrogen exhibits $s p^{2}$ character. The resonance of its axial lone pair with the phenyl ring is evidenced by the shortening of the bridge bond and by the almost parallel position of the phenyl ring and of the mean plane of the piperazine ring.
\end{abstract}

0108-2701/91/010227-03\$03.00
Experimental. White prisms, dimensions $0.20 \times 0.15$ $\times 0.08 \mathrm{~mm}$. Density not measured. Unit-cell parameters and intensity data obtained on an EnrafNonius CAD-4 diffractometer with graphitemonochromated $\mathrm{Cu} K \alpha$ radiation in $\omega / \theta$ scan mode $\left(0<\theta<65^{\circ}\right)$. Cell dimensions refined by leastsquares fitting of $\theta$ values of 25 reflections. No appreciable drop in intensity of two standard reflections $(\overline{2} 10,040)$ checked every 5400 s. 3081 independent reflections collected in $h, k, \pm l$, range $0,0,10$ to $10,26,10 ; 1360$ unique reflections with $I \geq 3 \sigma(I)$ used in subsequent calculations. Intensities corrected

(c) 1991 International Union of Crystallography 